\title{
Ginsparg-Wilson meson scattering on a staggered sea
}

\author{
Jiunn-Wei Chen, ${ }^{a}$ Donal O'Connell ${ }^{* b}$ and Andre Walker-Loud ${ }^{c}$ \\ ${ }^{a}$ Department of Physics, National Taiwan University, Taipei 10617, Taiwan \\ ${ }^{b}$ California Institute of Technology, 452-48, Pasadena, CA 91125, USA \\ ${ }^{c}$ Department of Physics, University of Maryland, College Park, MD 20742-4111 \\ Email: jwc@phys.ntu.edu.tw, donal@theory.caltech.edu, \\ walkloudeumd.edu
}

\begin{abstract}
We discuss the structure of the NLO corrections to the chiral formulae for mesonic scattering processes in mixed action simulations using Ginsparg-Wilson valence quarks and staggered sea quarks. In particular, we show that the analytic contribution of the NLO chiral Lagrangian is the same as in QCD. We also comment on how this result restricts the dependence of the amplitudes on the unknown parameter $C_{\mathrm{Mix}}$ appearing in the chiral theory appropriate for these systems. We conclude with some comments on the explicit scattering lengths.
\end{abstract}

XXIV International Symposium on Lattice Field Theory

July 23-28 2006

Tucson Arizona, USA

\footnotetext{
*Speaker.
} 


\section{Introduction}

In continuum quantum field theory in four dimensions, massless Dirac fermions respect chiral symmetry. This symmetry is crucial for our understanding of low energy processes of QCD: since the light quark masses are small compared to the QCD scale, one would expect to see signatures of chiral symmetry in the spectrum. Since these signatures are not observed, we assume that QCD dynamically breaks chiral symmetry at some scale $\Lambda_{\chi}$; this leads to concrete predictions which are phenomenologically successful. Consequently, one would like latticized QCD to enjoy some analogue of chiral symmetry. This is achieved if the quarks satisfy the Ginsparg-Wilson relation [1]; current realizations are domain wall fermions and overlap fermions. These quark discretizations are collectively known as Ginsparg-Wilson (GW) quarks.

Unfortunately GW quarks come with a price. Simulations using GW quarks are slower [2] than simulations with Wilson or staggered quarks. Staggered quarks, in particular, have been popular recently due to the availability of the MILC lattices [3]. But this discretization scheme has its own issues: staggered chiral perturbation theory $[4,5,6]$ is theoretically complicated, as demonstrated, for example, by the large number of operators in the NLO Lagrangian [7]. ${ }^{1}$

A practical compromise between the GW and staggered discretization schemes is to use staggered fermions for the sea quarks and GW fermions for the valence quarks[8]. This is particularly attractive since one can employ the MILC lattices, but as we shall see, it is also theoretically clean. Several recent simulations $[9,10,11,12,13,14]$ have utilized this kind of mixed action scheme, so it is important to understand the chiral extrapolation appropriate to these simulations.

Nevertheless, these mixed action simulations face their own challenges. Chiral perturbation theory for mixed action simulations (MA $\chi \mathrm{PT})[15,16]$ depends on a new, as yet undetermined parameter, $C_{\mathrm{Mix}}$. This parameter shifts the mass of mixed valence-sea mesons at tree level, so that for a meson with valence quark $v$ and sea quark $s$,

$$
m_{v s}^{2}=B\left(m_{v}+m_{s}\right)+a^{2} \Delta_{\text {Mix }}
$$

where $\Delta_{\text {Mix }}=16 C_{\text {Mix }} / f^{2}$ and $a$ is the lattice spacing. In addition, a mixed action theory violates unitarity at any finite lattice spacing, so one must take the continuum limit before unitarity can be restored, unlike a partially quenched theory. We will comment on these issues below.

Before we continue, let us introduce some notation. We shall denote our valence quarks as $u, d$ and $s$ while our sea quarks are $j, l$ and $r$. The leading order (LO) mass of a meson of quark content $a, b$ will be denoted $m_{a b}$. Meson masses which suffer a tree level lattice correction will be denoted with a tilde; for example, the mass of a meson with quark content $j, j$ is $\tilde{m}_{j j}^{2}=2 B m_{j}+a^{2} \Delta_{I}$, where $a^{2} \Delta_{I}$ is the mass shift of a taste identity meson due to the staggered potential. The next-to-leading order (NLO) pion mass will be denoted $m_{\pi}$ and similarly for the kaon mass. The leading order decay constant is denoted by $f$ while the NLO pion and kaon decay constants are $f_{\pi}$ and $f_{K}$, respectively.

\footnotetext{
${ }^{1} \mathrm{We}$ assume the validity of the fourth-root trick, though it has not been shown that the continuum limit of latticized QCD with rooted-staggered quarks is in the same universality class as QCD.
} 


\section{NLO Corrections}

Recently, the $\pi \pi$ scattering length was computed [17] in mixed action chiral perturbation theory (MA $\chi \mathrm{PT})$ for $\mathrm{GW}$ valence quarks on a staggered sea. In terms of leading order parameters, the scattering length is given by

$$
\begin{aligned}
a_{I=2}^{(0)}=- & \frac{m_{u u}}{8 \pi f^{2}}\left\{1+\frac{m_{u u}^{2}}{(4 \pi f)^{2}}\left[4 \ln \left(\frac{m_{u u}^{2}}{\mu^{2}}\right)+4 \frac{\tilde{m}_{j u}^{2}}{m_{u u}^{2}} \ln \left(\frac{\tilde{m}_{j u}^{2}}{\mu^{2}}\right)-1+l_{\pi \pi}^{\prime}(\mu)\right]\right. \\
& \left.\quad-\frac{m_{u u}^{2}}{(4 \pi f)^{2}}\left[\frac{\tilde{\Delta}_{P Q}^{4}}{6 m_{u u}^{4}}+\frac{\tilde{\Delta}_{P Q}^{2}}{m_{u u}^{2}}\left[\ln \left(\frac{m_{u u}^{2}}{\mu^{2}}\right)+1\right]\right]+\frac{\tilde{\Delta}_{P Q}^{2}}{(4 \pi f)^{2}} l_{P Q}^{\prime}(\mu)+\frac{a^{2}}{(4 \pi f)^{2}} l_{a^{2}}^{\prime}(\mu)\right\},
\end{aligned}
$$

where $\Delta_{P Q}^{2}=\tilde{m}_{j j}^{2}-m_{\pi}^{2}$. This result simplifies considerably [17] if one rewrites the scattering length in terms of the mass of the pion on the lattice and the decay constant of the pion measured on the lattice (we refer to these as lattice-physical parameters.) Then the scattering length becomes

$$
a_{I=2}^{(0)}=-\frac{m_{\pi}}{8 \pi f_{\pi}^{2}}\left\{1+\frac{m_{\pi}^{2}}{\left(4 \pi f_{\pi}\right)^{2}}\left[3 \ln \left(\frac{m_{\pi}^{2}}{\mu^{2}}\right)-1+l_{\pi \pi}(\mu)\right]-\frac{m_{\pi}^{2}}{\left(4 \pi f_{\pi}\right)^{2}} \frac{\tilde{\Delta}_{P Q}^{4}}{6 m_{\pi}^{4}}\right\} .
$$

It is worth commenting on some aspects of this simplification. In Eq. (2.2), the unknown counterterm which must be determined on the lattice, $l_{\pi \pi}$, is identical to the continuum, unquenched counterterm at NLO, while in Eq. (2.1) there are additional unknown unphysical terms $l_{P Q}^{\prime}$ and $l_{a^{2}}^{\prime}$. These unphysical effects must somehow be removed in the analysis of the lattice data. The expression Eq. (2.1) depends on the masses of the mixed valence-sea meson, $\tilde{m}_{j u}$. This mass depends on the unknown parameter $C_{\text {Mix }}$; therefore, this parameter must be determined if one wishes to extrapolate using Eq. (2.1). The lattice-physical expression Eq. (2.2), however, does not depend on this unknown parameter; in fact, the lattice-physical expression does not depend in any way on the mixed valence-sea mesons, and only differs from the continuum, unquenched scattering length by a known term which has its origin in the unitarity violating flavour neutral propagators. Our goal in this section is to explain why these simplifications occur, and to what extent we may expect similar simplifications in other mesonic processes.

To begin, let us consider the structure of the NLO Lagrangian of MA $\chi$ PT. This Lagrangian is determined by the symmetry structure of the underlying partially quenched, mixed action form of QCD, supplemented by the assumption that chiral symmetry is spontaneously broken. Since the theory has two sectors - the valence and sea sectors - it is worth considering the spurions arising from these sectors separately. In the valence sector, the quarks satisfy the Ginsparg-Wilson relation; therefore, they only explicitly violate chiral symmetry through the quark mass term. This is the same as in continuum, unquenched QCD and therefore the purely valence sector of the NLO chiral Lagrangian in the mixed action theory is the Gasser-Leutwyler Lagrangian. There are additional sources of chiral symmetry violation in the sea sector. In particular, for staggered sea quarks, there are additional spurions associated with taste symmetry violation at finite $a$. One might expect that these spurions would lead to $a$ dependent analytic terms in the NLO scattering amplitudes; however, we will now show that this does not occur.

At NLO in the power counting scheme appropriate to current simulations, the NLO operators in the chiral Lagrangian only contribute at tree level. Of course, all of the in and out states in a 
simulation involve valence quarks, so for the purposes of an NLO computation one can set the sea quarks equal to zero in the NLO operators. Since the spurions arising from the sea sector go to zero when the sea quarks go to zero, we see that at NLO the only relevant spurions are the purely valence spurions. However, we have seen above that the NLO purely valence Lagrangian coincides with the Gasser-Leutwyler Lagrangian, so no lattice spacing dependent analytic terms can arise in this way. An exception to this argument occurs for double-trace operators; however, these operators can only renormalize the parameters $f$ and $B$ occurring in the LO chiral Lagrangian. In lattice-physical parameters we eliminate $f$ and $B$ in favour of the decay constant measured on the lattice and the masses of the particles on the lattice; this removes any lattice spacing dependence arising from double trace operators. We conclude that there can never be any lattice-spacing dependence arising from NLO operators in mesonic scattering amplitudes, expressed in lattice-physical parameters, at next-to-leading order.

Let us consider some simple examples to help make these arguments more concrete. A list of the NLO operators appearing in the staggered chiral Lagrangian is given in [7]. Each of these operators also appears in the NLO Lagrangian describing mixed action chiral perturbation theory with staggered sea quarks, with the rule that the taste matrices $\xi$ of Ref. [7] are only non-zero on the sea quark subspace; this is equivalent to replacing $\Sigma \rightarrow P_{s} \Sigma P_{s}$, where $P_{s}$ is the projector to the sea subspace, in spurions associated with taste violation. For example, one operator in the Lagrangian is

$$
\mathscr{O}_{1}=a^{2} \operatorname{str}\left[\partial_{\mu} \Sigma^{\dagger} \partial^{\mu} \Sigma \xi_{5} P_{s} \Sigma^{\dagger} P_{s} \xi_{5} P_{s} \Sigma P_{s}\right]
$$

We only want to use this operator at tree level, so we should take the sea quarks to vanish; this sets $P_{s} \Sigma P_{s} \rightarrow 0$. Consequently, $\mathscr{O}_{1}$ does not contribute at NLO. For another example, consider the double trace operator

$$
\mathscr{O}_{2}=a^{2} \operatorname{str}\left[\partial_{\mu} \Sigma^{\dagger} \partial^{\mu} \Sigma\right] \operatorname{str}\left[\xi_{5} P_{s} \Sigma^{\dagger} P_{s} \xi_{5} P_{s} \Sigma P_{s}\right] .
$$

In this case, it helps to remember that $\Sigma$ is given in terms of the matrix of mesons $\Phi$ by $\Sigma=$ $\exp [2 i \Phi / f]$, so when we set the sea quarks to zero the trace over the sea subspace is just a constant, namely the number of sea quarks $N_{s}$. Thus, for the purposes of a next-to-leading order calculation $\mathscr{O}_{2}$ reduces to

$$
\mathscr{O}_{2} \rightarrow a^{2} \operatorname{str}\left[\partial_{\mu} \Sigma^{\dagger} \partial^{\mu} \Sigma\right]
$$

Notice that the effect of $\mathscr{O}_{2}$ is to introduce an $a^{2}$ shift to the leading order parameter $f$.

A similar argument applies to potential counterterms involving sea quark masses. These are associated with spurions arising from the sea sector and therefore they may not contribute to scattering amplitudes except to renomalise the parameters $f$ and $B$; this dependence is removed upon switching to lattice-physical parameters. However, there is another, perhaps more physical, way of seeing why the sea quark masses do not contribute to the scattering. To understand this, we must digress briefly on $\pi \pi$ scattering in $S U(3)$ chiral perturbation theory. Evidently, the strange quark mass $m_{s}$ is a parameter of $S U(3) \chi \mathrm{PT}$, and so one might think that the $\pi \pi$ scattering length includes analytic terms involving $m_{s}$. However, if we suppose that the strange quark is heavy and integrate it out, we must then recover $S U(2) \chi \mathrm{PT}$. Now, chiral symmetry forces any $m_{s}$ dependence in the analytic terms of the $\pi \pi$ amplitude to occur in the form $m_{\pi}^{2} m_{K}^{2}$. But the only counterterm in the on-shell $S U(2)$ scattering amplitude (Eq. (2.2) with $\tilde{\Delta}_{P Q}=0$ ) is proportional to $m_{\pi}^{4}$. It is not 
possible to absorb $m_{\pi}^{2} m_{K}^{2}$ into $m_{\pi}^{4}$, so there can be no $m_{s}$ dependence in the $S U(3) \pi \pi$ scattering amplitude. This is indeed the case [18].

Now, let us return to the situation with the valence-sea meson masses. For the purposes of this discussion, we can ignore the flavour-neutral and ghost sectors, reducing our theory from an $S U(6 \mid 3)$ theory to an $S U(6)$ theory. The sea quark dependence of this $S U(6)$ chiral perturbation theory is analogous to the $m_{s}$ dependence of $S U(3) \chi \mathrm{PT}$. A similar decoupling argument tells us that the sea quark masses cannot affect processes involving the valence sector provided one uses the analogues of on-shell parameters which are the lattice-physical parameters. We conclude that there can be no analytic dependence on the sea quark masses in a mesonic scattering amplitude. These rather abstract arguments have been verified by explicit computation [19].

To conclude this section, we have shown so far that there can be no dependence on the lattice spacing $a$ or the sea quark masses arising from the NLO operators in the MA $\chi$ PT Lagrangian at next-to-leading order in the chiral power counting. However, the scattering amplitudes can still depend on these quantities through loop corrections from the LO Lagrangian. Note that since there is no counterterm available to absorb divergences proportional to the lattice spacing or the sea quark masses; this is a restriction on the form of the loop corrections. In favourable cases this restriction is strong enough to remove all dependence on the valence-sea mesons as occurred in the $\pi \pi$ amplitude; this removes all dependence on the unknown parameter $C_{\mathrm{Mix}}$. In the next section we will discuss individual mesonic processes and their structure.

\section{Meson meson scattering}

In a forthcoming work [19] we present explicit expressions for the $K K$ and $K \pi$ scattering amplitudes at threshold. Here, we will restrict our comments to the dependence of these amplitudes on the valence-sea mesons. First, let us introduce some notation. It is useful to quantify the partial quenching in the mesonic sector with the parameters

$$
\begin{aligned}
\Delta_{j u}^{2} & =\tilde{m}_{j j}^{2}-m_{\pi}^{2}=2 B\left(m_{j}-m_{u}\right)+a^{2} \Delta_{I} \\
\Delta_{r s}^{2} & =\tilde{m}_{r r}^{2}-m_{s s}^{2}=2 B\left(m_{r}-m_{s}\right)+a^{2} \Delta_{I} .
\end{aligned}
$$

It is common to consider quantum theories which have unitary low energy sectors but which violate unitarity above some cutoff $\Lambda$. For example, $\phi^{4}$ theory with a Pauli-Villars regulator is simply a theory or a light self-interacting scalar with a heavy ghost field which violates unitarity. The parameters $\Delta$ which we have introduced are measures of unitarity violation in the low-energy sector of QCD; when these parameters are zero, for example, the effective theory in Minkowski space has cuts at the points expected by the optical theorem. From the point of view of the low energy theory, one would like to tune these parameters to zero to remove as much of the unitarity violation as possible. ${ }^{2}$ Note that this involves tuning the sea quarks to be lighter than the valence quarks; the high energy theory of quarks is non unitary but this is only seen above the non-perturbative scale of QCD, when perturbative computations are possible. With these ideas in hand, let us consider the mesonic scattering amplitudes.

\footnotetext{
${ }^{2}$ At higher orders, the $\Delta$ parameters will receive further corrections, but can generally be defined as the difference between lattice-physical sea-sea meson masses and lattice-physical valence-valence masses.
} 
The $K K$ scattering amplitude in lattice-physical parameters does not depend on the valencesea mesons and hence the unknown parameter $C_{\mathrm{Mix}}$. When $\Delta_{r s}=0$ the expression for the scattering amplitude is

$$
\begin{aligned}
\mathscr{M}= & -\frac{4 m_{K}^{2}}{f_{K}^{2}}+\frac{56 m_{K}^{4}}{9(4 \pi)^{2} f_{K}^{4}}-\frac{8 m_{K}^{4}}{(4 \pi)^{2} f_{K}^{4}} \ln \left(\frac{m_{K}^{2}}{\mu^{2}}\right)+\left(\frac{10 m_{K}^{2} m_{\pi}^{2}}{9(4 \pi)^{2} f_{K}^{4}}+\frac{2 m_{\pi}^{4}}{9(4 \pi)^{2} f_{K}^{4}}\right) \ln \left(\frac{m_{\pi}^{2}}{\mu^{2}}\right) \\
& -\left(\frac{m_{\pi}^{4}}{9(4 \pi)^{2} f_{K}^{4}}+\frac{64 m_{\pi}^{2} \tilde{m}_{X}^{2}}{45(4 \pi)^{2} f_{K}^{4}}-\frac{46 m_{K}^{2} \tilde{m}_{X}^{2}}{45(4 \pi)^{2} f_{K}^{4}}+\frac{13 \tilde{m}_{X}^{4}}{5(4 \pi)^{2} f_{K}^{4}}\right) \ln \left(\frac{\tilde{m}_{X}^{2}}{\mu^{2}}\right) \\
& -\frac{8\left(2 m_{K}^{2}+m_{\pi}^{2}\right)^{2}}{27(4 \pi)^{2} f_{K}^{4}\left(\tilde{m}_{X}^{2}-m_{\pi}^{2}\right)}\left(\tilde{m}_{X}^{2} \ln \left(\frac{\tilde{m}_{X}^{2}}{\mu^{2}}\right)-m_{\pi}^{2} \ln \left(\frac{m_{\pi}^{2}}{\mu^{2}}\right)\right)+\frac{m_{K}^{4}}{f_{K}^{4}} \ell_{G L}(\mu) \\
& +\frac{\tilde{\Delta}_{j u}^{2} \tilde{m}_{X}^{2}}{(4 \pi)^{2} f_{K}^{4}} \mathscr{F}_{1}\left(\frac{m_{\pi}^{2}}{\tilde{m}_{X}^{2}}, \frac{m_{K}^{2}}{\tilde{m}_{X}^{2}}, \frac{\tilde{m}_{X}^{2}}{\mu^{2}}\right)+\frac{\tilde{\Delta}_{j u}^{4}}{(4 \pi)^{2} f_{K}^{4}} \mathscr{F}_{2}\left(\frac{m_{\pi}^{2}}{\tilde{m}_{X}^{2}}, \frac{m_{K}^{2}}{\tilde{m}_{X}^{2}}, \frac{\tilde{m}_{X}^{2}}{\mu^{2}}\right) \\
& +\frac{\tilde{\Delta}_{j u}^{6}}{(4 \pi)^{2} f_{K}^{4} \tilde{m}_{X}^{2}} \mathscr{F}_{3}\left(\frac{m_{\pi}^{2}}{\tilde{m}_{X}^{2}}, \frac{m_{K}^{2}}{\tilde{m}_{X}^{2}}\right)+\frac{\tilde{\Delta}_{j u}^{8}}{(4 \pi)^{2} f_{K}^{4} \tilde{m}_{X}^{4}} \mathscr{F}_{4}\left(\frac{m_{\pi}^{2}}{\tilde{m}_{X}^{2}}\right),
\end{aligned}
$$

where the functions $\mathscr{F}_{i}$ are known functions [19]. Note that the counterterm $\ell_{G L}(\mu)$ in this expression is the same as the counterterm in the physical scattering amplitude in on-shell parameters as we expect from the discussion above.

The $K \pi$ scattering amplitude has a more complicated form than either the $\pi \pi$ or $K K$ scattering amplitudes. This arises from the fact that the $\pi$ and $K$ meson masses are not equal, which induces a net momentum flow through loop propagators in the $u$-channel. Consequently, in this case the scattering amplitude does depend on the valence-sea mesons and therefore on $C_{\mathrm{Mix}}$. The part of the $K \pi$ amplitude which depends on the sea quarks $F=j, l, r$ is

$$
\mathscr{M}_{v s}=\frac{1}{\left(4 \pi f^{2}\right)^{2}} m_{K} m_{\pi} \sum_{F}\left(C_{F d} \log \frac{\tilde{m}_{F d}^{2}}{\mu^{2}}-C_{F s} \log \frac{\tilde{m}_{F s}^{2}}{\mu^{2}}-2 m_{K} m_{\pi} J\left(\tilde{m}_{F d}^{2}\right)+4 m_{K} m_{\pi}\right)
$$

where

$$
\begin{aligned}
C_{F d} & =\frac{4 m_{K} m_{\pi}^{2}-\tilde{m}_{F d}^{2}\left(m_{K}+m_{\pi}\right)}{m_{K}-m_{\pi}} \\
C_{F s} & =\frac{4 m_{K}^{2} m_{\pi}-\tilde{m}_{F s}^{2}\left(m_{K}+m_{\pi}\right)}{m_{K}-m_{\pi}}
\end{aligned}
$$

and

$$
J(m)=4 \frac{\sqrt{m^{2}-m_{\pi}^{2}}}{m_{K}-m_{\pi}} \arctan \left[\frac{\left(m_{K}-m_{\pi}\right) \sqrt{m^{2}-m_{\pi}^{2}}}{m^{2}+m_{K} m_{\pi}-m_{\pi}^{2}}\right] .
$$

Although the amplitude does depend on the valence sea-mesons, it is straightforward to check that the $\mu$ dependence of the amplitude does not depend on the these mesons, in agreement with our comments above. Of course, the counterterm in the full $K \pi$ amplitude in lattice-physical parameters is given by the QCD expression in on-shell parameters.

\section{Conclusions}

It is well-known that simulations with Ginsparg-Wilson valence quarks have no lattice spacing dependence at tree level of chiral perturbation theory as a consequence of the chiral properties of 
GW quarks. We have shown that this persists at next-to-leading order in the chiral expansion for mesonic scattering processes, expressed in lattice-physical parameters, in the sense that the unknown analytic term which must be determined from the lattice, does not depend on the lattice spacing. In addition, we have shown that there is no unknown analytic terms involving the sea meson masses. This situation is favourable because it simplifies the extraction of the interesting physical information from a lattice simulation of these processes: in principle, one need only use one lattice spacing, for example.

In addition, we have commented on the dependence of the $K K$ and $K \pi$ scattering amplitudes on the unknown parameter $C_{\text {Mix }}$ which appears in the leading order chiral Lagrangian describing mixed action processes. The $K K$ scattering amplitude does not depend on this parameter, but the $K \pi$ amplitude does; therefore, correct chiral extrapolation of $K \pi$ scattering data requires the determination of this parameter.

\section{References}

[1] P. H. Ginsparg and K. G. Wilson, Phys. Rev. D 25, 2649 (1982).

[2] A. D. Kennedy, Nucl. Phys. Proc. Suppl. 140, 190 (2005) [arXiv:hep-lat/0409167].

[3] C. W. Bernard et al., Phys. Rev. D 64, 054506 (2001) [arXiv:hep-lat/0104002].

[4] W. J. Lee and S. R. Sharpe, Phys. Rev. D 60, 114503 (1999) [arXiv:hep-lat/9905023].

[5] C. Aubin and C. Bernard, Phys. Rev. D 68, 034014 (2003) [arXiv:hep-lat/0304014].

[6] C. Aubin and C. Bernard, Phys. Rev. D 68, 074011 (2003) [arXiv:hep-lat/0306026].

[7] S. R. Sharpe and R. S. Van de Water, Phys. Rev. D 71, 114505 (2005) [arXiv:hep-lat/0409018].

[8] D. B. Renner et al. [LHP Collaboration], "Hadronic physics with domain-wall valence and improved staggered sea Nucl. Phys. Proc. Suppl. 140, 255 (2005) [arXiv:hep-lat/0409130].

[9] S. R. Beane, P. F. Bedaque, K. Orginos and M. J. Savage [NPLQCD Collaboration], Phys. Rev. D 73, 054503 (2006) [arXiv:hep-lat/0506013].

[10] R. G. Edwards et al. [LHPC Collaboration], Phys. Rev. Lett. 96, 052001 (2006) [arXiv:hep-lat/0510062].

[11] S. R. Beane, K. Orginos and M. J. Savage, arXiv:hep-lat/0604013.

[12] S. R. Beane, K. Orginos and M. J. Savage, arXiv:hep-lat/0605014.

[13] S. R. Beane, P. F. Bedaque, K. Orginos and M. J. Savage, arXiv:hep-lat/0606023.

[14] S. R. Beane, P. F. Bedaque, T. C. Luu, K. Orginos, E. Pallante, A. Parreno and M. J. Savage, arXiv:hep-lat/0607036.

[15] O. Bar, G. Rupak and N. Shoresh, Phys. Rev. D 67, 114505 (2003) [arXiv:hep-lat/0210050].

[16] O. Bar, C. Bernard, G. Rupak and N. Shoresh, Phys. Rev. D 72, 054502 (2005) [arXiv:hep-lat/0503009].

[17] J. W. Chen, D. O'Connell, R. S. Van de Water and A. Walker-Loud, Phys. Rev. D 73, 074510 (2006) [arXiv:hep-lat/0510024].

[18] M. Knecht, B. Moussallam, J. Stern and N. H. Fuchs, Nucl. Phys. B 457, 513 (1995) [arXiv:hep-ph/9507319].

[19] J. W. Chen, D. O'Connell and A. Walker-Loud, in preparation. 